\title{
ON THE FREQUENCIES OF TYPE I AND TYPE II SUPERNOVAE
}

\author{
( Abstract)
}

\author{
N. DA LLA PORTA \\ Istituto di Astronomia, Universita di Padova, Italia
}

I would like to present some simple considerations concerning the frequency of supernovae of both types and the mass range of their stellar progenitors which I have tried to deduce from the statistics given by Tammann (1970) for Sb and Sc galaxies only, and from some other data of other authors (Bertola and Sussi, 1965; Barbon, 1968) I will just briefly describe the procedure I have followed.

Relying on the data of a fiducial period from 1959 to 1969, Tammann gives the frequency of occurrence of supernovae per unit mass $\left(10^{10} M_{\odot}\right)$; in order to separate type I and type II, I have first statistically divided the unidentified cases according to the observed ratio of type I/type II. As it has been observed that type II SN occur only in Sb, Sc and Irregular galaxies, they may be considered as belonging entirely to young Population I. The frequency of type II SN is then obtained by dividing the observed number of events by the number of galaxies considered in the survey, times the average mass young population I content of $\mathrm{Sb}$ and $\mathrm{Sc}$ galaxies (from 7 to $10 \%)$. The mean frequency per unit mass $\left(10^{10} M_{\odot}\right)$, per young population I and per 100 years is given by:

$\begin{array}{ll}\mathrm{Sb} & \mathrm{Sc} \\ 1.09 & 1.57\end{array}$

All supernovae appearing in elliptical and So galaxies are of type I. However, many of them are also in spirals; and moreover their location in spirals shows that some of them are located in the central bulk and other in the spiral arms. There is thus some indication that type I SN might be a mixture of two different kinds of events, with similar external phenomenology, but belonging to objects of quite different populations, which one may term as SNIo (old) and SNIy (young). Separating again statistically the observed SNI events according to the observed ratio from the location in Spirals (Bertola and Sussi, 1965) of SNIo/SNIy, we calculate the frequencies of both SNIo and SNIy by dividing the numbers of events thus obtained by the number of galaxies considered and the mean mass amounts of old disk population (76 to $67 \%$ ) and respectively of young disk Population I ( 7 to $10 \%$ ). We thus arrive at the following figures for the mean frequencies per unit mass $\left(10^{10} M_{\odot}\right)$ and per 100 years and per population type

$\begin{array}{lll} & \mathrm{Sb} & \mathrm{Sc} \\ \text { SNIy } & 0.25 & 1.2 \\ \text { SNIo } & 0.007 & 0.058\end{array}$


The number of SNI observed in elliptical and So galaxies (Barbon, 1968) appears to be compatible with the assumption that they are the same kind of events as the SNIo in spirals.

If now one further assumes the Salpeter birthrate function as being representative also for the young disk Population I of Sb and Sc galaxies, and that death rates equal birthrates, it is then easy to calculate the mass range of SNIy and SNII progenitors yielding the observed frequencies. One finds the following values, for the lower limits of visual, bolometric absolute magnitudes and mass of the progenitors:

\begin{tabular}{lll} 
& SNII & SNIy \\
\hline$M_{v}$ & -3.7 & -4.0 \\
$M_{b}$ & -5.7 & -6.0 \\
Mass & $\sim 13 M_{\odot}$ & $\sim 15 M_{\odot}$
\end{tabular}

Should the observed frequencies reported by Tammann be somewhat underestimated due to losses, the mass range of the progenitors could perhaps be lowered to some $\sim 10 M_{\odot}$. In any way, this value obtained is higher than those generally quoted.

\section{References}

Barbon, R.: 1968, Astron. J. 73, 1016.

Bertola, F. and Sussi, M. G.: 1965, Contrib. Oss. Astr. Univ. Padova, No. 176.

Tammann, G. A.: 1970, Astron. Astrophys. 8, 458. 